PROCEEDINGS OF THE

AMERICAN MATHEMATICAL SOCIETY

Volume 124, Number 12, December 1996, Pages 3889-3896

S 0002-9939(96)03628-3

\title{
VASSILIEV INVARIANTS OF TYPE TWO FOR A LINK
}

\author{
HITOSHI MURAKAMI
}

(Communicated by Ronald Stern)

\begin{abstract}
We show that any type two Vassiliev invariant of a link can be expressed as a linear combination of the second coefficients of the Conway polynomials of its components and a quadratic expression of linking numbers.
\end{abstract}

V.A. Vassiliev introduced a concept of finite type invariants for knots [9] by studying the cohomology of the space of all knots. J.S. Birman and X.-S. Lin gave its combinatorial definition [3]. It turns out that finite type (or Vassiliev) invariants form a wide family of knot invariants including quantum invariants (see for example [2]). For other properties including M. Kontsevich's integral formula, see [1].

Vassiliev invariants can be naturally generalized to links [7]. For a knot, it is well known that the only type one Vassiliev invariant is a constant. On the other hand it is easily shown that the linking number of any pair of components in a link is a Vassiliev invariant of type one. We will show that every Vassiliev invariant of type one is a sum of a constant and a linear combination of linking numbers.

For a type two Vassiliev invariant, we will show that it can be expressed as a sum of the $z^{2}$-coefficients of the Conway polynomials of its components and linking numbers. As a corollary we can express the second derivative of the Jones polynomial at one in terms of the Conway polynomials and linking numbers, which was proved in [6] without using the concept of Vassiliev invariants.

\section{Preliminaries}

In this section we describe terminology used in this paper. For more detail we refer the reader to $[2,3]$.

For a (complex-valued) link invariant $v_{d}$, we regard it as an invariant for spatial 4-valent knotted graphs (or singular links) as follows:

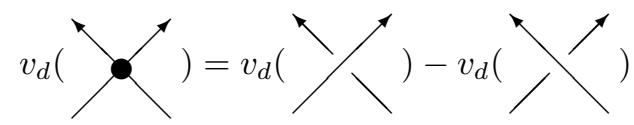

If the graph invariant defined above vanishes for all graphs with more than $d$ vertices, then we call $v_{d}$ a Vassiliev invariant of type $d$. This is equivalent to saying

Received by the editors March 15, 1995.

1991 Mathematics Subject Classification. Primary 57M25.

Key words and phrases. Vassiliev invariant, Conway polynomial.

Partially supported by Grant-in-Aid for Scientific Research on Priority Area 231 "Infinite Analysis", the Ministry of Education, Science and Culture, Japan. 
that $v_{d}$ is constant on all the graphs with the same $[d]$-configuration. Here a $[d]$ configuration is $d$ pairs of $2 d$ points on disjoint circles. For example,

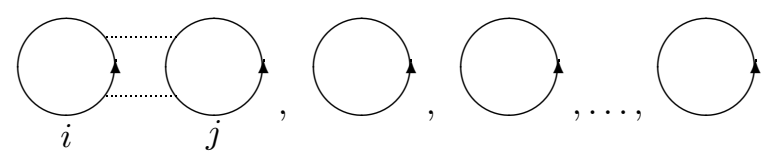

is a [2]-configuration, where dotted lines indicate the pairing. A singular link $G$ with $d$ vertices respects a $[d]$-configuration if each set of paired points forms a vertex in $G$. The following singular link respects the [2]-configuration above:

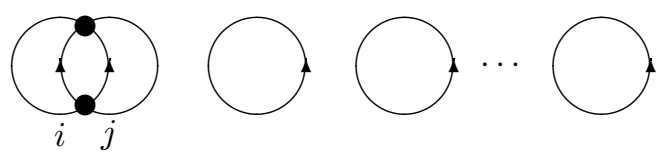

Suppose that we are given a Vassiliev invariant of type $d$ for $n$-component ordered, oriented links. As is described in [3], if we know all the initial data (values of singular links respecting [e]-configurations $(e \leq d)$, where singular links are chosen one for each configuration, and for $[d]$-configurations the initial data does not depend on singular links respecting it), then we can calculate the value of every link using $(*)$. This proves the following lemma.

Lemma 1.1. Two Vassiliev invariants of the same type coincide if they have the same initial data.

\section{VASSILIEV INVARIANTS OF TYPE ONE}

In this section, we will study Vassiliev invariants of type one for an $n$-component link. For a 1-component link (a knot), it is well known (see [3]) that a constant is the only Vassiliev invariant of type one.

Let $L=L_{1} \cup L_{2} \cup \cdots \cup L_{n}$ be an ordered, oriented, $n$-component link. Denote by $\lambda_{i j}(L)$ the linking number of $L_{i}$ and $L_{j}(i<j)$. Then we easily see the following lemma.

Lemma 2.1. $\lambda_{i j}$ is a Vassiliev invariant of type one.

Proof. We show that $\lambda_{i j}$ is constant on all the singular links with one vertex

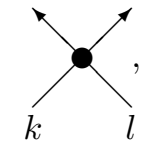

where $k$ and $l$ mean the label attached to the strands nearby. We may assume $k \leq l$ without loss of generality. If $k=l$, which corresponds to the following [1]-configuration:

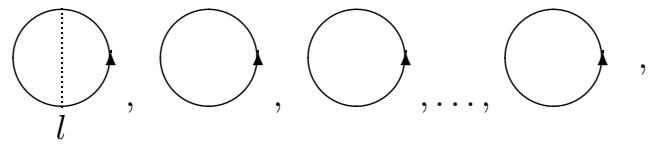

then

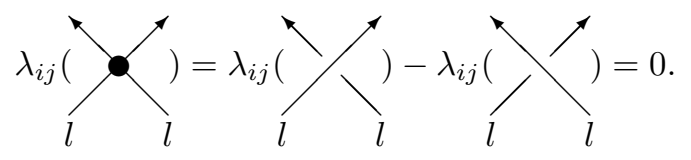


If $k<l$, which corresponds to the following [1]-configuration:

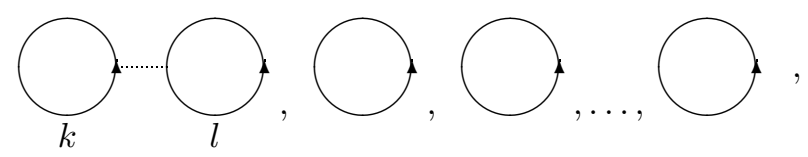

then

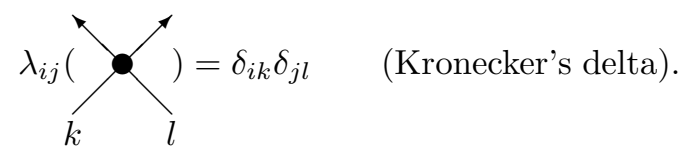

So $\lambda_{i j}\left(C_{l}\right.$ ) depends only on [1]-configurations (i.e., it can be expressed in terms

of $i, j, k$, and $l$ ) and $\lambda_{i j}$ is a Vassiliev invariant of type one.

As a corollary, we have

Corollary 2.2. Fix an integer $n \geq 1$. Let $a$ and $b_{i j}(1 \leq i<j \leq n)$ be constants (depending only on $n$ ). For an n-component link $L$, put

$$
w_{1}(L)=a+\sum_{i<j} b_{i j} \lambda_{i j}(L)
$$

Then $w_{1}$ is a Vassiliev invariant of type one.

Now suppose that we are given another Vassiliev invariant $v_{1}$ of type one. We want to choose $a$ and $b_{i j}$ to make $v_{1}$ and $w_{1}$ equal. To use Lemma 1.1, we need to list all the admissible $[i]$-configurations and singular links respecting them $(0 \leq i \leq 1)$. (A configuration with no paired adjacent points on the same circle is called admissible. Note that since we can choose a model graph for an inadmissible configuration so that its Vassiliev invariant vanishes, we need not consider inadmissible configurations [3].) There are two types of such configurations.

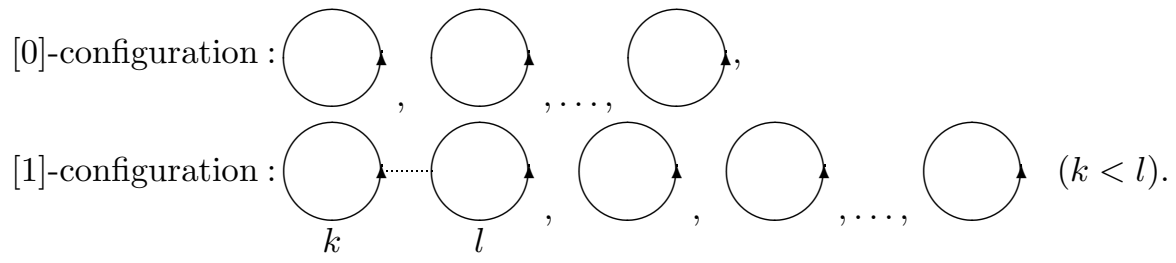

For the [0]-configuration we choose the $n$-component trivial link $O^{n}$ as a model graph. Then the initial data of $v_{1}$ and $w_{1}$ are as follows. (Note that we do not specify a model graph of the [1]-configuration.)

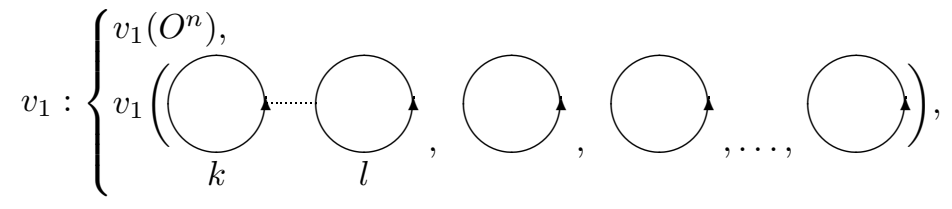


and

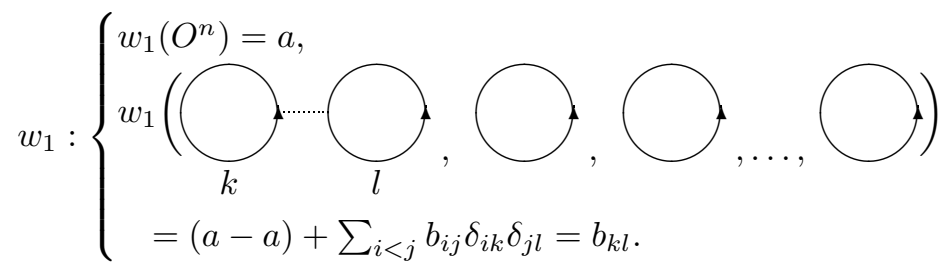

From Lemma 1.1 we have the following theorem.

Theorem 2.3. Let $v_{1}$ be a Vassiliev invariant of type one for $n$-component ordered, oriented links. Then for any $n$-component ordered, oriented link $L$ we have

$$
+\sum_{i<j} v_{1}\left(\bigcirc_{i}(L)=v_{1}\left(O^{n}\right)\right.
$$

Proof. From the initial data above, if we choose

$$
a=v_{1}\left(O^{n}\right)
$$

and

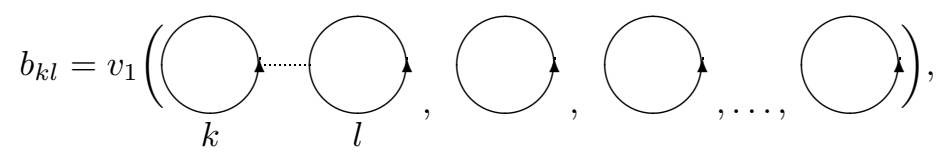

then $v_{1}$ and $w_{1}$ coincide by virtue of Lemma 1.1. The proof is complete.

\section{VASSILIEV INVARIANTS OF TYPE TWO}

In the proof of Theorem 2.3, it is essential that the number of admissible $[i]$ configurations $(i \leq 1)$ is equal to that of "linearly independent" type one Vassiliev invariants. In this section, we will use a similar fact to describe how to express a Vassiliev invariant of type two in terms of the $z^{2}$-coefficients of the Conway polynomials of the components and the linking numbers.

It is well known (see $[1,4,5])$ that if $v_{d}$ and $v_{e}$ are Vassiliev invariants of types $d$ and $e$ respectively, then $v_{d} v_{e}$ (i.e., $v_{d} v_{e}(L)=v_{d}(L) v_{e}(L)$ for every link $\left.L\right)$ is of type $d+e$. So we have the following lemma.

Lemma 3.1. Put $\lambda_{i j} \lambda_{k l}(L)=\lambda_{i j}(L) \lambda_{k l}(L)$. We also denote $\lambda_{i j} \lambda_{i j}$ by $\lambda_{i j}^{2}$. Then $\lambda_{i j} \lambda_{k l}$ is a Vassiliev invariant of type two. Here $i<j, k<l$ and $i \leq k$.

Since the coefficient of $z^{2}$ of the Conway polynomial of a knot is a Vassiliev invariant of type two [1], there are six types of type two Vassiliev invariants; constants, $\varphi_{i}(1 \leq i \leq n), \lambda_{i j}(i<j), \lambda_{i j}^{2}(i<j), \lambda_{i j} \lambda_{i k}(j<k)$, and $\lambda_{i j} \lambda_{k l}(i<j, k<l, i<k)$, where $\varphi_{i}$ is the $z^{2}$-coefficient of the Conway polynomial of the $i$-th component. So we have the following corollary. 
Corollary 3.2. Fix an integer $n(\geq 1)$. Let $a, b_{i}, c_{i j}, d_{i j}, e_{i j k}, f_{i j k l}$ be constants (depending only on $n$ ). Then

$$
w_{2}=a+\sum_{i=1}^{n} b_{i} \varphi_{i}+\sum_{i<j} c_{i j} \lambda_{i j}+\sum_{i<j} d_{i j} \lambda_{i j}^{2}+\sum_{\substack{i \\ j<k}} e_{i j k} \lambda_{i j} \lambda_{i k}+\sum_{\substack{i<j \\ k<l \\ i<k}} f_{i j k l} \lambda_{i j} \lambda_{k l}
$$

is a Vassiliev invariant of type two for n-component links.

Now there are four types of admissible [2]-configurations:
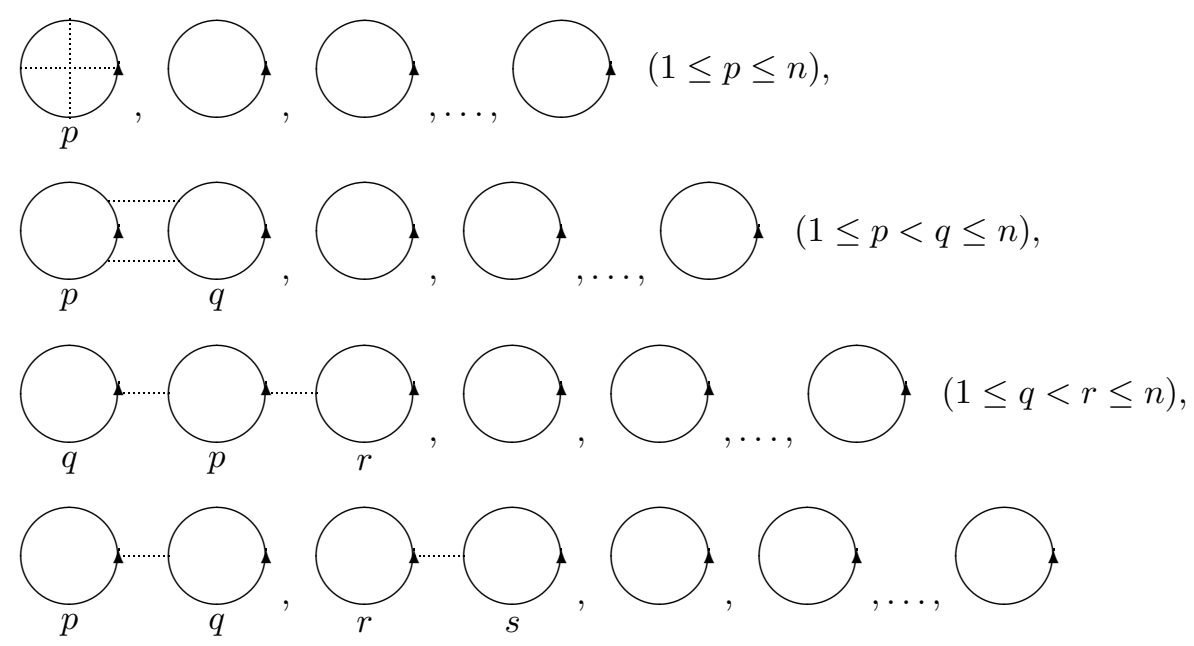

$$
(1 \leq p<q \leq n, 1 \leq r<s \leq n, p<r) .
$$

Together with [0]- and [1]-configurations, we see that the number of admissible $[i]$-configurations $(i \leq 2)$ is equal to that of (possibly linearly dependent) type two Vassiliev invariants. We can show that these Vassiliev invariants are linearly independent. In fact, we can prove the following theorem.

Theorem 3.3. Let $v_{2}$ be a Vassiliev invariant of type two for $n$-component ordered, oriented links. Then for any n-component ordered, oriented link $L$, we have

$$
+\sum_{i=1}^{v_{2}(L)=v_{2}\left(O^{n}\right)}
$$




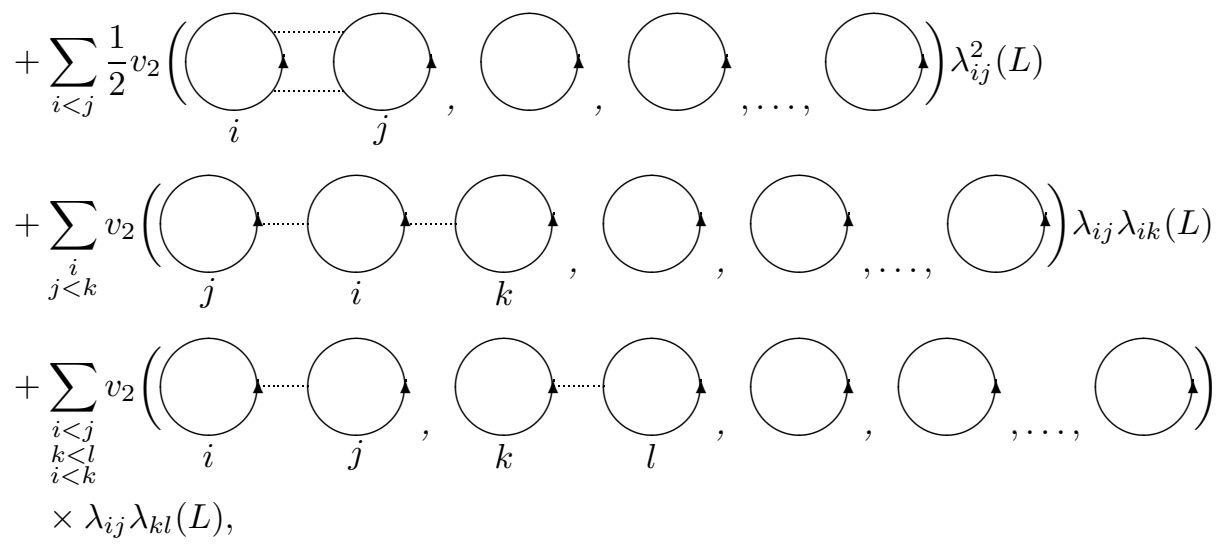

where $\circ$ denotes the split union (disjoint union separated by a 2-sphere).

Proof. The proof is similar to that of Theorem 2.3. We will choose $a, b_{i}, c_{i j}, d_{i j}$, $e_{i j k}$, and $f_{i j k l}$ so that the initial data for $v_{2}$ and $w_{2}$ coincide.

Choosing model graphs $O^{n}$ and $\left(C_{p}\right) O^{n-2}$ for the [0]-configuration

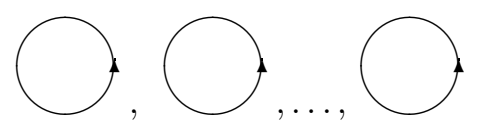

and the [1]-configuration

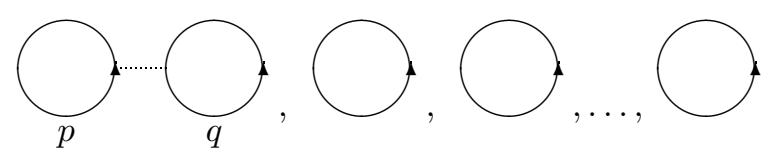

respectively, we can list below the initial data for $w_{2}$. Note that

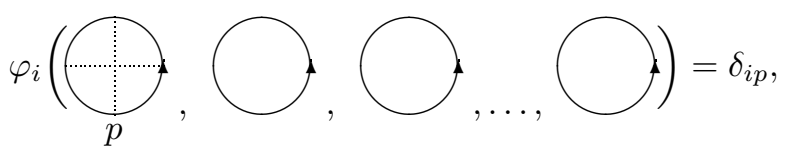

since $\left.\varphi_{1}()_{1}\right)$ is equal to the $z^{2}$-coefficient of the Conway polynomial of the trefoil knot [3].

$$
w_{2}(\underbrace{w_{2}}_{p}\left(O^{n}\right)=a,
$$



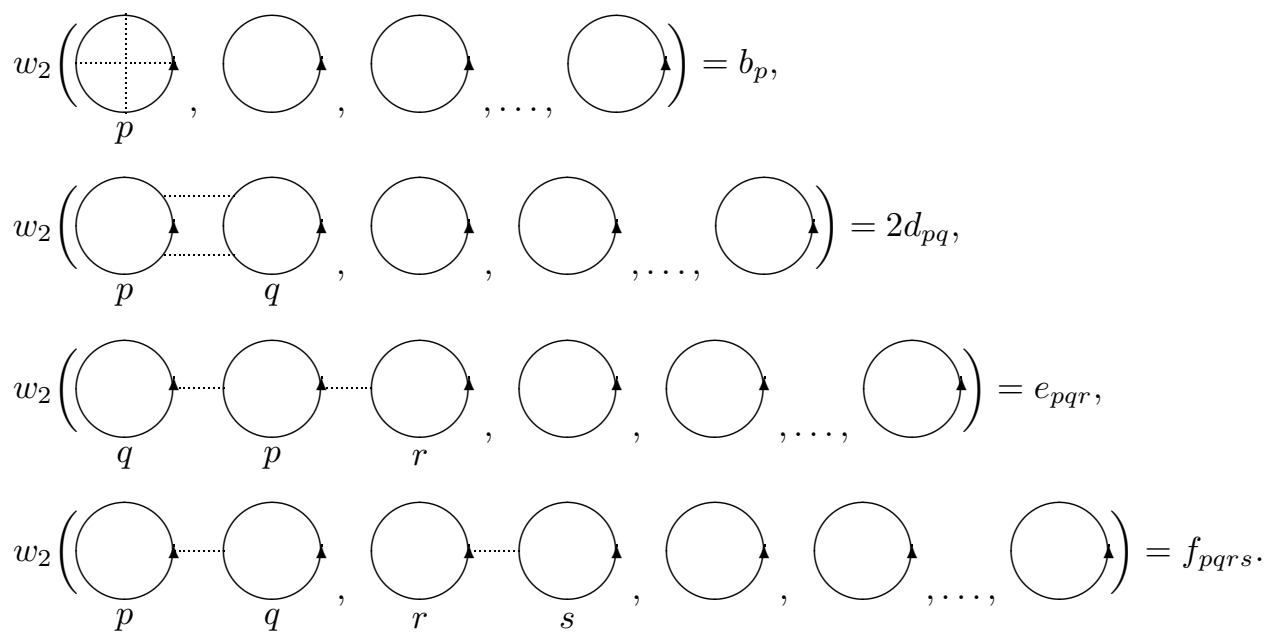

Now the only thing we have to do is to solve a system of linear equations. Details are left to the reader.

As a corollary, we can express the second derivative of the Jones polynomial at one in terms of the $z^{2}$-coefficients of the Conway polynomials of the constituent knots and linking numbers which was proved in [6] since it is a Vassiliev invariant of type two (see for example [3]).

Remark 3.4. In the proofs of Theorems 2.3 and 3.3, it happens that the number of admissible configurations equals the number of (linearly independent) Vassiliev invariants. Y. Miyazawa [5] and A. Tani [8] proved that this also holds for type three invariants and gave a similar formula. The author does not know whether this holds for more general cases.

\section{ACKNOWLEDGEMENTS}

The result here was prepared for a talk at the Isaac Newton Institute for Mathematical Sciences, Cambridge in the autumn of 1992. The author thanks R. Kirby for encouraging him to give a talk. Thanks are also due to the Institute for hospitality.

\section{REFERENCES}

[1] D. Bar-Natan, On the Vassiliev knot invariant, Topology 34 (1995), 423-472. CMP 95:08

[2] J.S. Birman, New points of view in knot theory, Bull. Amer. Math. Soc. (N.S.) 28 (1993), 253-287. MR 94b:57007

[3] J.S. Birman and X.-S. Lin, Knot polynomials and Vassiliev's invariants, Invent. Math. 111 (1993), 225-270. MR 94d:57010

[4] T. Kanenobu and Y. Miyazawa, Link polynomials as Vassiliev-type invariants, preprint, Osaka City Univ. and Yamaguchi Univ., 1994.

[5] Y. Miyazawa, Vassiliev's invariant and link polynomials (in Japanese), Teijigen-Tayotai no Toporojii to Musubime-riron (Topology of Low-dimensional Manifolds and Knot Theory), Proceedings of Research Institute for Mathematics and Computer Science, vol. 9, Tsuda College, 1994.

[6] H. Murakami, On derivatives of the Jones polynomial, Kobe J. Math. 3 (1986), 61-64. MR 88a:57015

[7] T. Stanford, Finite-type invariants of knots, links, and graphs, to appear in Topology. 
[8] A. Tani, Vassiliev type invariant of order 3 (in Japanese), Teijigen-Tayotai no Toporojii to Musubime-riron (Topology of Low-dimensional Manifolds and Knot Theory), Proceedings of Research Institute for Mathematics and Computer Science, vol. 9, Tsuda College, 1994.

[9] V.A. Vassiliev, Cohomology of knot spaces, Theory of Singularities and Its Applications (V.I. Arnold, ed.), Advances in Soviet Math., vol. 1, Amer. Math. Soc., 1990. MR 92a:57016

Department of Mathematics, Osaka City University, Sugimoto, Sumiyoshi-ku, Osaka 558 , JAPAN

Current address: Department of Mathematics, School of Science and Engineering, Waseda University, Ohkubo, Tokyo, 169, Japan

E-mail address: hitoshi@haya.co.jp 